\title{
Pilot study of single-session radiofrequency ablation of benign thyroid nodules in Singapore
}

\section{Dear Editor,}

Radiofrequency ablation (RFA) has been shown to be safe and efficacious in the treatment of benign thyroid nodules. ${ }^{1}$ Ion agitation during RFA generates high temperatures between 60 and 100 degree Celsius to cause tissue damage and size reduction. ${ }^{2}$

We conducted a prospective pilot study of 10 patients who underwent thyroid RFA between September 2016 and December 2018. Inclusion criteria included benign thyroid nodules that cause symptoms such as neck pain, dysphagia, foreign body sensation and cosmetic issues; benign autonomously functioning thyroid nodules causing thyrotoxicosis; and nodules that were clearly visualised on ultrasound (US) imaging. Exclusion criteria included nodules of less than $60 \%$ solid component, nodules that were poorly visualised on US imaging, subjects younger than 21 years of age, presence of a pacemaker in subjects, underlying coagulopathy, poor skin condition, known thyroid carcinoma, and allergy to local anaesthetic.

All patients underwent 2 separate nodule sampling by US-guided fine needle aspiration cytology and/or core needle biopsy. Only nodules that were proven to be benign on 2 samples were ablated. Complications were documented with reference to the Common Terminology Criteria for Adverse Events V4.0. They included, but were not limited to, minor complications such as pain, bleeding, haematoma formation, and major complications such as skin burns, voice change and injury to surrounding neck structures.

Physical examination and US imaging were obtained at baseline, 2 weeks, 6 months and 12 months after a single RFA procedure. Nodule volume reduction, cosmetic and symptom scores were assessed. Nodule volume was calculated using the equation: $V=\pi a b c / 6$ ( $V=$ volume, $a=$ maximum diameter, $b$ and $c=$ the other 2 perpendicular diameters). The size, shape, margin, proportion of solid/cystic components, echogenicity, calcification, internal vascularity, and extracapsular invasion of each nodule were recorded. Nodules with malignant US features were excluded. The cosmetic score: $1=$ no palpable mass, $2=$ palpable mass, $3=$ mass visible on swallowing, $4=$ readily detected cosmetic problem; and symptom score (visual analogue scale of 0-10) were obtained at every visit. Therapeutic success was defined as a volume reduction of $>50 \%$ at 12 months, a benchmark used by other thyroid RFA studies. ${ }^{3-5}$ Baseline laboratory tests, which included complete blood count, coagulation profile and thyroid function tests, were performed. Incidentally, all the treated nodules were cold nodules.

RFA procedures were conducted by interventional radiologists. The moving shot technique, proposed by Baek et al., ${ }^{4}$ was used. Grounding pads were applied to the patient's thigh. RFA was performed using the STARmed RF system (STARmed, Goyang, South Korea), which consists of a VIVA RF generator and electrode. The danger triangle, consisting of the recurrent laryngeal nerve and oesophagus, was avoided. After the procedure, a post-treatment US appearance of the thyroid nodule and complications were recorded. The patients were discharged after at least 1 hour of observation.

There were 8 female and 2 male patients. The mean age was 43 years (interquartile range [IQR] 36.3-50.5). All patients had benign histology ( 8 patients had nodular goitres and 2 had colloid nodules) (Table 1). Each patient underwent a single RFA session for a single thyroid nodule. The mean ablation time and power were 9.7 minutes (IQR 6.1-14.3) and 5.7kCal (IQR 3.4-8.1), respectively. Seven out of 10 patients met the criteria for therapeutic success (volume reduction of $>50 \%$ at 12 months). The median pre-ablation nodule volume was $18.1 \mathrm{~mL}$ (IQR 9.7-26.4) and the median nodule composition was $85 \%$ solid (IQR 67.5-92.5). Mean and median nodule volume reduction were $44.4 \%$ and 57.1\% (IQR 7.4-67.4), respectively at 12 months. Two out of the 3 patients who did not achieve a volume reduction of $>50 \%$ had a 3 -point reduction in their cosmetic scores still. There was a median cosmetic score reduction of 3 points (IQR 2-3). Patients who were symptomatic pre-treatment had a median symptom score reduction of 1 point (IQR 1-4). All patients indicated that they would recommend the procedure to other patients.

All complications were minor. All patients complained of varying degrees of pain, but it did not warrant stoppage of the RFA procedures. Four patients had minor bleeding and/or haematoma formation that 


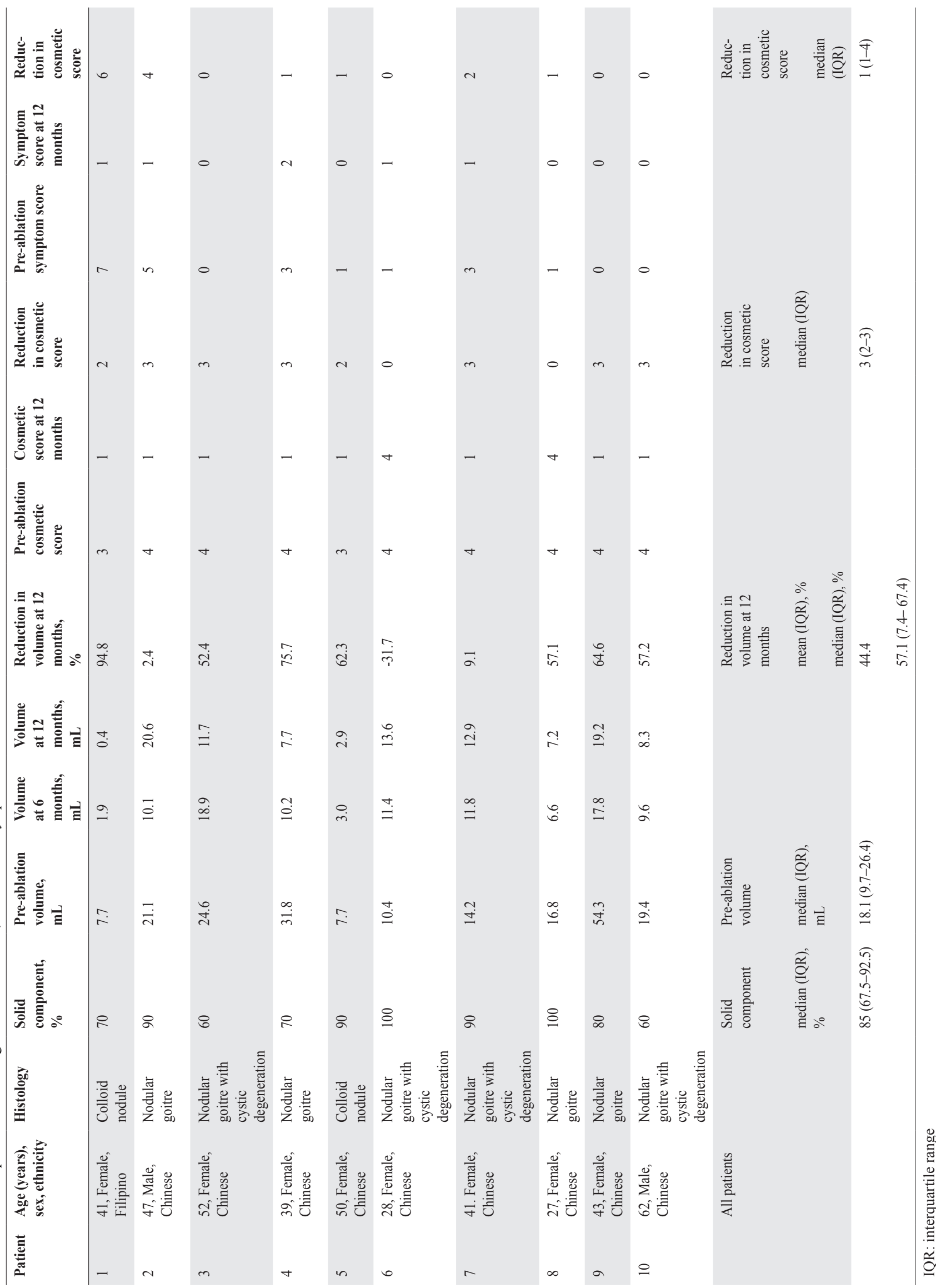


resolved 2 weeks post-procedure. There was no major complication.

The results of our study were similar to a 2018 study by Hamidi et al. $^{3}$ that achieved a median volume reduction of $44.6 \%$ (IQR 42.1-59.3). This was most likely due to the similarities in nodule profiles (predominantly solid and large nodules) and that only a single ablation session was conducted in both studies. In contrast, other studies with single ablation attempts by Baek et al. ${ }^{4}$ and Faggiano et al. ${ }^{5}$ achieved higher mean volume reduction of $82.6 \%$ and $84.9 \%$, respectively. We postulate that this may be related to their lower pre-ablation nodule volumes of $7.5 \mathrm{~mL}$ (standard deviation [SD] 4.9) and $13.3 \mathrm{~mL}$ (SD 1.8), respectively. Other studies that achieved a higher volume reduction had multiple

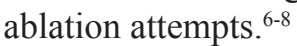

The limitations of our study are a small study number and the lack of long-term data. Further larger and longerterm studies are needed to evaluate the efficacy and safety of single-session RFA of benign thyroid nodules in the Singapore population. The result of this pilot study suggests that RFA of benign thyroid nodules is safe and efficacious for treating predominantly solid benign thyroid nodules in the Singapore population.

\section{REFERENCES}

1. Chung SR, Suh $\mathrm{CH}$, Baek JH, et al. Safety of radiofrequency ablation of benign thyroid nodules and recurrent thyroid cancers: a systematic review and meta-analysis. Int J Hyperthermia 2017; 33:920-30.

2. Goldberg SN. Radiofrequency tumor ablation: principles and techniques. Eur J Ultrasound 2001;13:129-47.
3. Hamidi O, Callstrom MR, Lee RA, et al. Outcomes of radiofrequency ablation therapy for large benign thyroid nodules: A Mayo Clinic case series. Mayo Clin Proc 2018;93:1018-25.

4. Baek JH, Kim YS, Lee D, et al. Benign predominantly solid thyroid nodules: prospective study of efficacy of sonographically guided radiofrequency ablation versus control condition. AJR Am J Roentgenol 2010;194:1137-42.

5. Faggiano A, Ramundo V, Assanti AP, et al. Thyroid nodules treated with percutaneous radiofrequency thermal ablation: a comparative study. J Clin Endocrinol Metab 2012;97:4439-45.

6. Cheng Z, Che Y, Yu S, et al. US-guided percutaneous radiofrequency versus microwave ablation for benign thyroid nodules: A prospective multicenter study. Sci Rep 2017;7:9554.

7. Dobnig H, Amrein K. Monopolar radiofrequency ablation of thyroid nodules: A prospective Austrian single-center study. Thyroid 2018;28:472-80.

8. Huh JY, Baek JH, Choi H, et al. Symptomatic benign thyroid nodules: efficacy of additional radiofrequency ablation treatment session--prospective randomized study. Radiology 2012;263:909-16.

Elvin YT Lim, ${ }^{1}$ FRCR, Sum Leong, ${ }^{2}$ FFR RCSI,

Harold HW Heah, ${ }^{3}$ MMed (ORL), Jeremy CF Ng, ${ }^{4}$ FRCSEd (Gen), Chiaw Ling $\underline{\text { Chng, }}{ }^{5}{ }_{M R C P}(U K)$, Chow Wei Too, ${ }^{2}{ }_{F R C R}$

${ }^{1}$ Department of Radiology, Changi General Hospital, Singapore

${ }^{2}$ Department of Vascular and Interventional Radiology, Singapore General Hospital, Singapore

${ }^{3}$ Department of Otolaryngology, Singapore General Hospital, Singapore

${ }^{4}$ Department of General Surgery, Singapore General Hospital, Singapore

${ }^{5}$ Department of Endocrinology, Singapore General Hospital, Singapore

Correspondence: Dr Elvin Lim, Department of Radiology, Changi General Hospital, 2 Simei Street 3, Singapore 529889.

Email: elvin.lim.y.t@singhealth.com.sg 